\title{
Vaccination with recombinant NetB toxin partially protects broiler chickens from necrotic enteritis
}

\author{
Anthony L Keyburn ${ }^{1,2,3^{*}}$, Ricardo W Portela ${ }^{1,4}$, Kathy Sproat ${ }^{1}$, Mark E Ford ${ }^{1}$, Trudi L Bannam ${ }^{2,3}$, Xuxia Yan², \\ Julian I Rood ${ }^{2,3}$ and Robert J Moore 1,2,3 $^{1,2,3}$
}

\begin{abstract}
NetB toxin from Clostridium perfringens is a major virulence factor in necrotic enteritis in poultry. In this study the efficacy of NetB as a vaccine antigen to protect chickens from necrotic enteritis was examined. Broiler chickens were immunized subcutaneously with purified recombinant NetB (rNetB), formalin treated bacterin and cell free toxoid with or without rNetB supplementation. Intestinal lesion scores and NetB antibody levels were measured to determine protection after mild oral gavage, moderate in-feed and heavy in-feed challenges with virulent $C$. perfringens isolates. Birds immunized with rNetB were significantly protected against necrotic enteritis when challenged with a mild oral dose of virulent bacteria, but were not protected when a more robust challenge was used. Bacterin and cell free toxoid without rNetB supplementation did not protect birds from moderate and severe in-feed challenge. Only birds immunized with bacterin and cell free toxoid supplemented with rNetB showed significant protection against moderate and severe in-feed challenge, with the later giving the greatest protection. Higher NetB antibody titres were observed in birds immunized with rNetB compared to those vaccinated with bacterin or toxoid, suggesting that the in vitro levels of NetB produced by virulent C. perfringens isolates are too low to induce the development of a strong immune response. These results suggest that vaccination with NetB alone may not be sufficient to protect birds from necrotic enteritis in the field, but that in combination with other cellular or cell-free antigens it can significantly protect chickens from disease.
\end{abstract}

\section{Introduction}

Necrotic enteritis in chickens is a common bacterial disease that costs the global poultry production industry an estimated US $\$ 2$ billion annually [1]. The causative agent is the bacterium Clostridium perfringens. Currently, ionophore anticoccidials or antibiotic growth promoters are used to control necrotic enteritis [2]. However, the risk of antibiotic resistance and consumer pressure has prompted the industry to reduce the use of in-feed antibiotics and it is likely that the use of ionophore anticoccidials will also be reduced. In the European Union, the use of most antibiotic growth promotants has been banned, and necrotic enteritis remains an ongoing issue for producers in these countries $[3,4]$. This situation has increased the need to develop other

\footnotetext{
* Correspondence: anthony.keyburn@csiro.au

${ }^{1}$ CSIRO Biosecurity Flagship, Australian Animal Health Laboratory, Geelong 3220, Australia

${ }^{2}$ ARC Centre of Excellence in Structural and Functional Microbial Genomics, Department of Microbiology, Monash University, Clayton 3800, Australia Full list of author information is available at the end of the article
}

methods to control necrotic enteritis in poultry. Vaccination is an alternative approach that could be deployed to manage necrotic enteritis in the absence of antibiotics and anticoccidials.

Vaccines against other clostridial diseases in production animals have been widely and successfully used for many years and are based on protection from specific toxins produced by the bacteria that are associated with the particular disease [5]. Necrotic enteritis in chickens is a notable exception; it is an economically important clostridial disease for which there are limited vaccines available. Although necrotic enteritis has been recognised as a significant clostridial disease of chickens for 50 years [6], progress towards the development of a vaccine has been very limited until recently. Historically, C. perfringens alpha-toxin was implicated as the major virulence factor in the disease, which led to vaccine development efforts based around this toxin. Several experimental vaccines based on alpha-toxin have been reported and they have had variable protective success [7-9]. However, an alpha-
C Biomed Central

(c) 2013 Keyburn et al.; licensee BioMed Central Ltd. This is an Open Access article distributed under the terms of the Creative Commons Attribution License (http://creativecommons.org/licenses/by/2.0), which permits unrestricted use, distribution, and reproduction in any medium, provided the original work is properly cited. 
toxin deficient mutant strain of $C$. perfringens has been shown to retain full virulence [10], indicating that the toxin is not an essential virulence factor. Despite this observation it is clear that antibodies raised against this toxin can partially protect birds from disease. Although alpha-toxin is a secreted protein, Zekarias et al. [9] have shown that some of the protein remains associated with the cell membrane. It is presumably immune interaction with this cell-associated protein that provides the protective effect seen with some alpha-toxin based vaccines. The fact that vaccines using live attenuated alpha-toxin negative strains of $C$. perfringens are effective against avian necrotic enteritis [11] demonstrates that there must be other antigens of $C$. perfringens that are capable of inducing a protective immune response. Some of these protective antigens have been identified in recent studies $[12,13]$.

Recently, a secreted $\beta$-pore forming toxin, NetB, has been isolated from a virulent chicken isolate of $C$. perfringens and shown to be essential for disease induction [14]. NetB toxin has been found in most $C$. perfringens isolates from necrotic enteritis-diseased birds, but is uncommon in isolates recovered from healthy birds [15-17]. As an important virulence factor, NetB represents an attractive vaccine candidate, as shown in a recent study where vaccination with NetB induced some protection of broiler birds against experimental necrotic enteritis [18]. The studies reported here not only test whether NetB can be used as a protective vaccine antigen as a single subunit vaccine, but investigate whether NetB in combination with other antigenic proteins, either whole cell bacterin or secreted $C$. perfringens toxoid, can enhance protection in chickens against necrotic enteritis.

\section{Materials and methods Strains}

C. perfringens strains EHE-NE18 [10] and WER-NE36 were used as challenge strains in the in vivo necrotic enteritis disease induction models described below. EHENE18 (Type A, net $B^{+}$) has been previously shown [10] to be virulent in an in vivo model while WER-NE36 (Type $A$, net $B^{+}$) was isolated recently from a clinical case and shown to be highly virulent in our disease models.

\section{Vaccine preparation and delivery}

A single EHE-NE18 colony from a Tryptose Sulphite Cycloserine agar plate (TSC agar, Oxoid, Basingstoke, United Kingdom) was inoculated into $20 \mathrm{~mL}$ of Trypticase-peptone-glucose (TPG) medium and grown overnight at $37^{\circ} \mathrm{C}$. Ten $\mathrm{mL}$ of the resultant culture were inoculated into one litre of TPG medium and grown at $37^{\circ} \mathrm{C}$ to an $\mathrm{OD}_{600 \mathrm{~nm}}$ of $0.8-1.0$. The culture was centrifuged at $6000 \mathrm{~g}$ for $10 \mathrm{~min}$ at $4^{\circ} \mathrm{C}$, filtered through a $0.45 \mu \mathrm{m}$ membrane and concentrated by ultrafiltration through a $10 \mathrm{kDa}$ membrane (Merck Millipore, Massachusetts, USA) to $40 \mathrm{~mL}(25 \times)$. The bacterial cells were resuspended in $40 \mathrm{~mL}$ of phosphate buffered saline (PBS; $137 \mathrm{mM} \mathrm{NaCl}, 10 \mathrm{mM}$ phosphate, $2.7 \mathrm{mM}$ $\mathrm{KCl}, \mathrm{pH}$ 7.4) and sonicated (three times for $30 \mathrm{~s}$ ) to disrupt the cellular membranes and release cytoplasmic proteins. Formaldehyde (40\%) was added to both the concentrated supernatant and the cells to a final concentration of $0.3 \%(\mathrm{v} / \mathrm{v})$. Bacterin comprised 50:50 (v/v) bacterial cells and culture supernatant, while toxoid consisted of formaldehyde-treated culture supernatant only.

Recombinant NetB (rNetB) was expressed and purified (98\% purity) as previously described [14] and formalin treated as described above. Fifty $\mu \mathrm{g}$ of rNetB per bird was used for NetB subunit, bacterin- and toxoidsupplemented vaccines. Each bird was vaccinated subcutaneously at day 7 and day 17 using CSIRO triple adjuvant [19] (comprising 60\% (v/v) Montanide, 40\% (v/v) antigen combined with Quil A, $3 \mathrm{mg} / \mathrm{mL}$, and DEAEdextran, $30 \mathrm{mg} / \mathrm{mL}$ in PBS) in a total volume of $500 \mu \mathrm{L}$.

\section{Necrotic enteritis disease induction models}

Two different challenge methods were used: a direct oral gavage challenge with small volumes of culture, which may be more akin to challenges that occur in the field, and a large volume in-feed challenge that gives a more robust challenge, resulting in higher levels of disease in a greater percentage of birds. These oral gavage and infeed necrotic enteritis disease induction models were performed essentially as previously described [10,20], with some modification to the timing to fit in with vaccination schedules. Briefly, groups of 10 chickens were kept in adjacent, but separate, pens in an animal isolation facility. Commercial 1-day-old Ross 308 broiler chickens were fed an antibiotic-free chicken starter diet containing 20\% (w/w) protein for 22 days. On the morning of day 23 the feed was changed to a high protein wheat-based feed containing 50\% fishmeal. On day 28 , chickens were euthanized with inhaled carbon dioxide and their small intestines (duodenum to ileum) examined for gross necrotic lesions. Intestinal lesions in the small intestine were scored as before [10]: $0=$ no gross lesions; 1 = thin or friable walls; 2 = focal necrosis or ulceration (1-5 foci); 3 = focal necrosis or ulceration (615 foci); 4 = focal necrosis or ulceration (16 or more foci); 5 = patches of necrosis $2-3 \mathrm{~cm}$ long; 6 = diffuse necrosis typical of field cases. The statistical significance of differences between groups was assessed using Kruskal-Wallis one-way ANOVA. All animal experiments were assessed, approved and monitored by the Australian Animal Health Laboratory's Animal Ethics Committee. Experimental models of C. perfringens 
infection to induce necrotic enteritis require the addition of predisposing factors. The most widely published models either use high protein feed or Eimeria infection. Eimeria infection can cause immunological stress, which may be inappropriate in a vaccine study. Therefore, we chose to use high protein as the predisposing factor in this study.

\section{Oral gavage challenge}

For the oral gavage challenge, C. perfringens strain EHENE18 was grown in fluid thioglycollate broth (FTG; Difco) with the addition of $2 \%(\mathrm{w} / \mathrm{v})$ soluble starch and $1.5 \%(\mathrm{w} / \mathrm{v})$ thiopeptone and incubated at $37^{\circ} \mathrm{C}$ for $14 \mathrm{~h}$. On the evening of day 24 the feed was withdrawn and each bird was orally challenged with $1.5 \mathrm{~mL}$ of $C$. perfringens culture $\left(10^{9}\right.$ to $\left.10^{10} \mathrm{CFU}\right)$. On day 25 birds were again orally challenged and feed contaminated with C. perfringens $(1: 10 \quad(\mathrm{v} / \mathrm{w})$ culture to feed) was administered.

\section{In-feed challenge}

For the in-feed challenge, 1-2 C. perfringens (EHE-NE18 or WER-NE36) colonies grown on TSC agar were transferred into $10 \mathrm{~mL}$ of cooked meat medium (CMM; Difco Becton Dickinson, Maryland, USA) and incubated at $37^{\circ} \mathrm{C}$ for $18 \mathrm{~h}$. One $\mathrm{mL}$ of the resulting culture was used to inoculate $20 \mathrm{~mL}$ of FTG, and, after incubation at $37^{\circ} \mathrm{C}$ for $18 \mathrm{~h}, 1 \mathrm{~mL}$ was used to inoculate $20 \mathrm{~mL}$ of CMM and incubated at $37^{\circ} \mathrm{C}$ for $18 \mathrm{~h}$. Twenty $\mathrm{mL}$ of the final CMM culture was used as the inoculum for $800 \mathrm{~mL}$ of FTG medium, and after $18 \mathrm{~h}$ incubation at $37^{\circ} \mathrm{C}$, the culture was mixed with feed for the challenge. A separate serially passed culture was prepared for each challenge feeding $(n=4)$. High protein-feed and FTG culture were mixed in a ratio of $3: 4(\mathrm{v} / \mathrm{w})$. The mixture was then placed into feed trays. Birds were fed the culture/feed mix twice a day on days 26 and 27. Trays were cleaned and the remaining feed discarded prior to each subsequent feeding.

\section{Experimental design}

For NetB immunogenic studies two groups of 10 birds were challenged in-feed (described above) with either EHE-NE18 or WER-NE36. Blood samples from the wing (brachial vein) were taken and serum collected directly before challenge and 6 and 10 days post challenge. Serum anti-NetB IgY levels were determined by enzymelinked immunosorbent assay (ELISA).

The vaccine studies were performed with the same vaccination regime but separated into three different challenge models; (i) oral gavage challenge with EHENE18 to produce mild disease, (ii) in-feed challenge using EHE-NE18 to produce moderate levels of disease, and (iii) an in-feed challenge with WER-NE36 to produce the most severe disease. For vaccine studies a blood sample was taken from each bird, immediately following euthanasia, for serum analysis by ELISA and Western blotting.

\section{Western blotting and immunoassay detection of antibodies}

NetB-specific antibody levels were determined by the end-point dilution method using an ELISA. Microtiter plates (Nunc Maxsorp, Roskilde, Denmark) were coated with $\mathrm{rNetB}$ protein $(1 \mu \mathrm{g} / \mathrm{mL}$ in $0.1 \mathrm{M}$ sodium carbonate buffer, $\mathrm{pH}$ 9.6) for $16 \mathrm{~h}$ at $4^{\circ} \mathrm{C}$. The coated plates were washed twice with PBS (pH 7.6) and blocked for $3 \mathrm{~h}$ at room temperature with $5 \%(\mathrm{w} / \mathrm{v})$ bovine serum albumin (BSA; Sigma Aldrich, Missouri, USA) in PBS (PBSB). The plates were washed twice with PBS and sera from the immunized birds, and the concurrent control groups, diluted 1:400 in 1\% BSA, were added and the plates incubated for $1 \mathrm{~h}$ at $37^{\circ} \mathrm{C}$. The plates were washed 5 times with PBS plus Tween $20(0.05 \%(\mathrm{v} / \mathrm{v})$, PBST) and once with PBS before the addition of goat-antichicken IgY horseradish peroxidase conjugate (KPL, Maryland, USA) diluted 1:2000 in PBSB and incubated for $1 \mathrm{~h}$ at $37^{\circ} \mathrm{C}$. After extensive washing of the plates with PBST $(5 \times)$ and PBS $(1 \times)$ the colour reaction was developed by using a tetramethylbenzidine alkaline phosphate substrate kit (Invitrogen), following the manufacturer's instructions. The reactions were stopped by the addition of $50 \mu \mathrm{L}$ of $0.5 \mathrm{M} \mathrm{NaOH}$ and the absorbance at $450 \mathrm{~nm}$ was measured in a microplate spectrophotometer (BioTek). The specific antibody level of the immune serum was expressed as the $A_{450}$ value above the cut-off [21], which was defined as the mean absorbance value of the unimmunized plus three standard deviations.

A recombinant NetB preparation was run on SDSPAGE (NuPAGE ${ }^{\circ}$ Novex 4-12\% Bis-Tris gel, Invitrogen) in MES SDS buffer (NuPAGE ${ }^{\circ}$ MES SDS Running Buffer, Invitrogen, California, USA). Protein was transferred onto PDVF (Invitrogen) membrane and probed with immune serum from vaccinated birds. Blots were developed with an ECL Western Blotting kit (Amersham Biosciences, GE Life Sciences, Bunkinghamshire, United Kingdom) and the results recorded on autoradiographic film.

\section{Results}

\section{NetB is immunogenic in chickens}

To investigate whether in vivo produced native NetB was able to invoke an immune response during an infection, groups of 10 birds were challenged separately, infeed (see material and methods for details), with two different virulent $C$. perfringens strains, EHE-NE18 and WER-NE36, and allowed to recover from the infection. 
Serum collected at pre-infection, 6 and 10 days postchallenge, was used in a NetB-specific ELISA. Pooled serum from birds challenged with either EHE-NE18 or WER-NE36, showed strong anti-NetB antibodies, with the highest levels observed 6 days post challenge (Figure 1). These results demonstrated that NetB stimulates an immune response in birds that have been challenged with virulent strains.

\section{rNetB induces a protective immune response}

To determine the immunogenicity of purified rNetB, chickens were immunized at day 7 , boosted at day 17 , and oral gavage challenged with EHE-NE18 at days 24 and 25. Serum was collected at day 28 post-hatch. These sera were tested for NetB specific antibodies using Western blot analysis (Figure 2) and ELISA (Figure 3a). Chickens immunized with $\mathrm{rNetB}$ had significantly higher levels of anti-NetB IgY antibodies $(p<0.001)$ than birds treated with adjuvant alone or the no treatment controls. Western blot analysis using sera from the rNetB vaccinated birds showed strong antibody binding to $r N e t B$ (33 kDa).

To determine if vaccination with $\mathrm{rNetB}$ can protect birds from disease, birds were immunised with rNetB and then challenged with pathogenic $C$. perfringens. Protection was assessed by the average lesion score of the treatment groups. The rNetB vaccine provided statistically significant $(p<0.05)$ protection against an oral gavage $C$. perfringens challenge in comparison to the adjuvant and no treatment control groups (Figure 4a). There was a $58 \%$ reduction in the number of birds with lesions and a significant reduction in the average lesion score $(65 \%$ reduction, $p<0.05)$ when the rNetBvaccinated group was compared with the combined adjuvant and no treatment groups.

A second, more aggressive in-feed, homologous challenge model was used to further characterise the

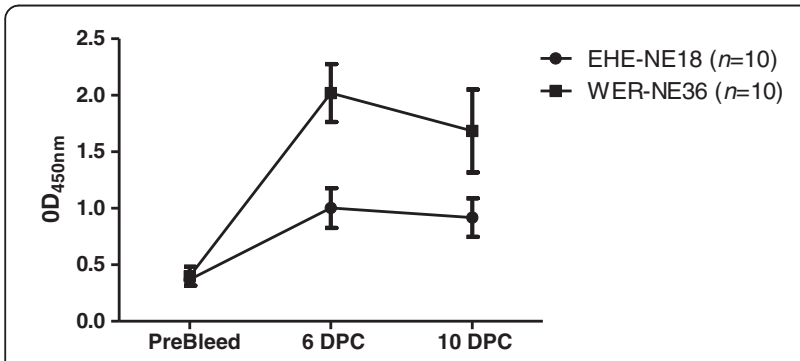

Figure 1 NetB specific lgY production in birds inoculated with two different $\boldsymbol{C}$. perfringens strains. Two groups of 10 birds were challenged in-feed (days 26 and 27) either with EHE-NE18 (•) or WER-NE36 (- and serum collected on days 26 (pre-bleed), 33 (6 days post-challenge, 6 DPC) and 37 (10 days post challenge, 10 DPC). The levels of serum IgY antibodies against NetB were measured by ELISA and expressed as the average optical density at $450 \mathrm{~nm}$. The error bars represent SEM.

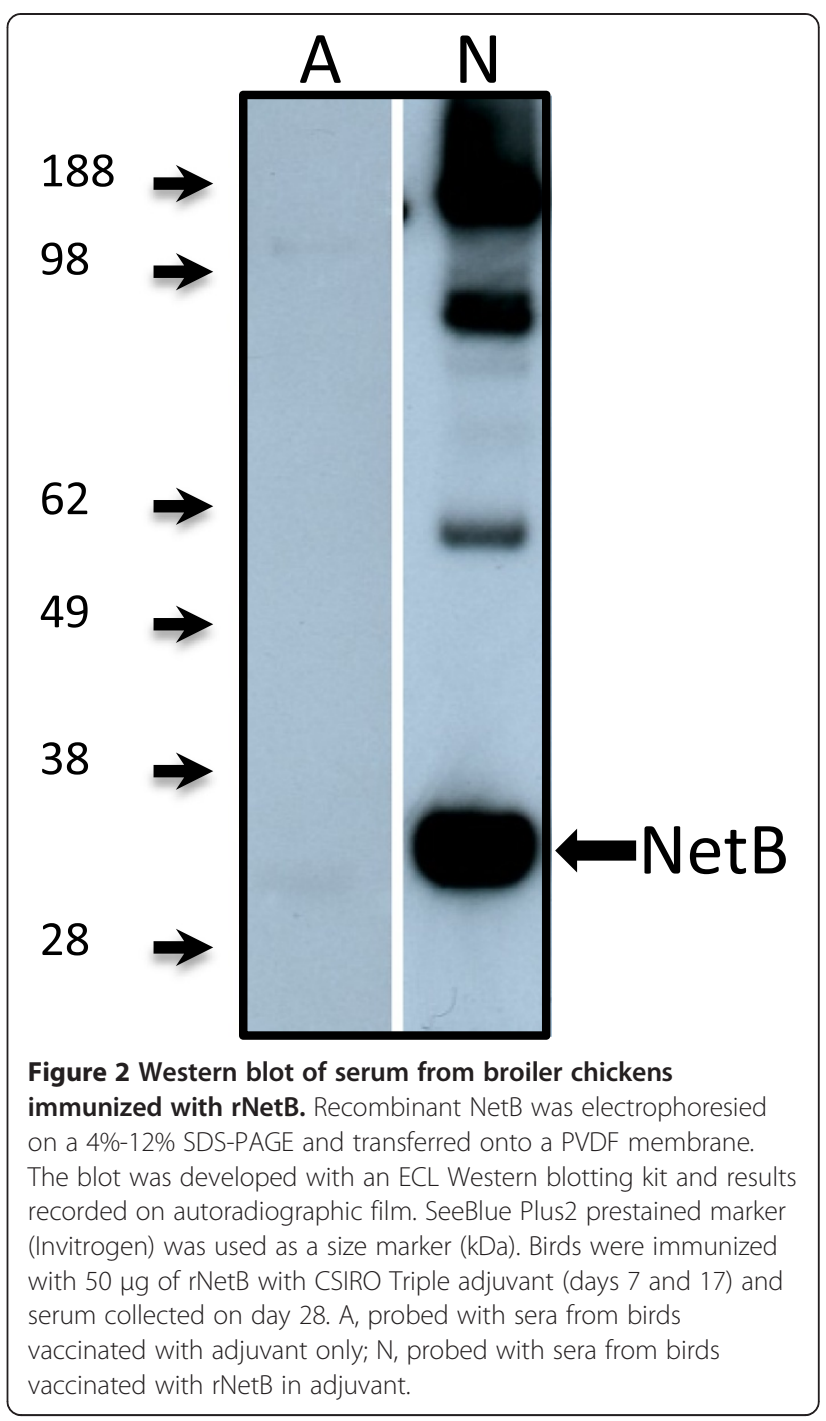

protective efficacy of the $\mathrm{rNetB}$ vaccine. To determine if improved efficacy could be obtained we also immunised other birds with either whole cell bacterin or whole cell bacterin supplemented with rNetB. The birds immunised with $\mathrm{rNetB}$ or bacterin alone were not protected against this more aggressive homologous challenge (Figure $4 \mathrm{~b}$ ). However, birds immunised with bacterin supplemented with $\mathrm{rNetB}$ had a statistically significant reduction in lesion scores compared to both the negative control groups $(p<0.05)$, with $67 \%$ reduction in birds with lesions and $76 \%$ reduction in average lesion score. Birds immunised with rNetB alone (Figure $3 \mathrm{~b}$ ) had the highest level of antiNetB IgY antibodies whereas birds immunised with bacterin had very low levels. Those birds immunised with bacterin supplemented with $\mathrm{rNetB}$ had significantly higher $(p<0.05)$ levels of anti-NetB IgY antibodies compared to the no treatment control birds.

To determine if bacterin supplemented with rNetB could protect against a heterologous challenge we 

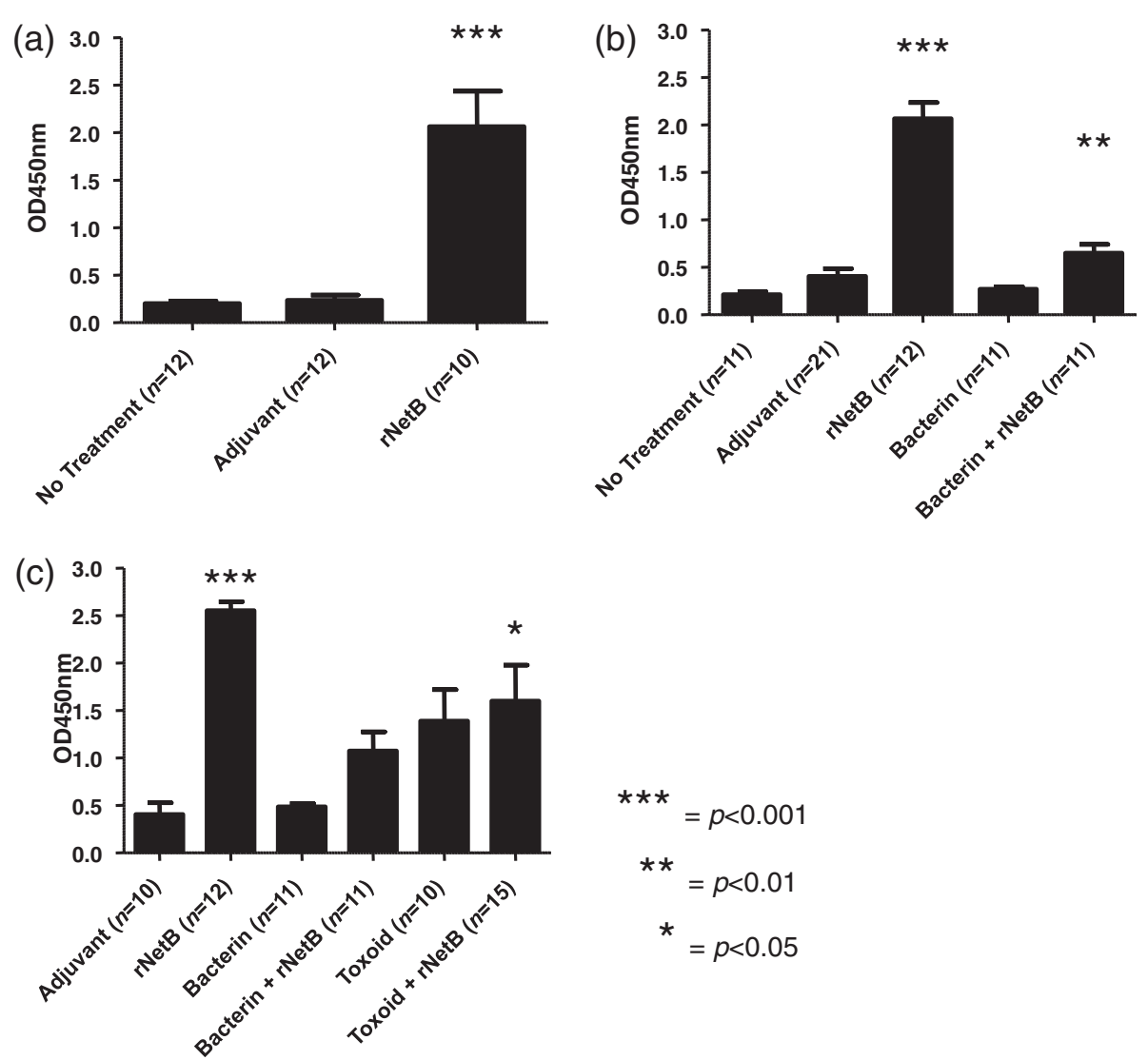

Figure 3 Serum IgY responses of broiler chickens immunized with clostridial proteins. Chickens were immunized subcutaneously with the indicated vaccines on days 7 and 17, challenged with C. perfringens, and the levels of serum IgY antibodies against NetB were measured by ELISA at day 28. Adjuvant only and no treatment groups were used as controls. (a) Birds were immunized and challenged orally with EHE-NE18 (days 24 and 25). (b) Birds were immunized and challenged in-feed with EHE-NE18 (days 26 and 27). (c) Birds were immunized and challenge in-feed with WER-NE36 (days 26 and 27). The error bars represent SEM.

immunised birds with $\mathrm{rNetB}$, bacterin and bacterin supplemented with rNetB. We also immunised chickens with toxoid (cell free inactive proteins) and toxoid supplemented with rNetB. The birds were challenged, in-feed, with the most highly virulent strain available to us, WER-NE36. Those birds immunised with either rNetB or bacterin showed no reduction in lesion score compared to the adjuvant control group (Figure 4c). The group immunised with toxoid had a lower average disease score but was not statistically significantly protected $(p>0.05)$. Only chickens immunised with bacterin or toxoid supplemented with $\mathrm{rNetB}$ showed statistically significant $(p<0.05)$ decreases in lesion score compared to the adjuvant control group (Figure $4 \mathrm{c}$ ), with the rNetBsupplemented toxoid vaccine reducing the number of birds with lesions by $67 \%$ and the average lesion score by $74 \%$. Birds immunised with $\mathrm{rNetB}$ alone had the highest levels of anti-NetB IgY antibodies (Figure 3c). Similar to the previous vaccination trial, chickens immunised with bacterin had very low NetB-specific antibody levels while the group vaccinated with bacterin supplemented with $\mathrm{rNetB}$ had higher levels compared to the adjuvant control group. Birds immunised with toxoid or toxoid with rNetB had similar levels of anti-NetB IgY antibody (Figure 3c) although only toxoid with $\mathrm{rNetB}$ was significantly higher $(p<0.05)$ compared to the adjuvant control group.

\section{Discussion}

This study has evaluated whether NetB, a major virulence factor in necrotic enteritis, is an effective protective antigen when used as a subunit vaccine or as a supplement to either traditional bacterin or toxoid vaccines. rNetB alone was protective against a mild challenge with a virulent $C$. perfringens strain, but was not sufficient to protect against a heavy in-feed challenge. Immunisation with bacterin or cell free toxoid supplemented with $\mathrm{rNetB}$ significantly protected birds against necrotic enteritis following a heavy challenge with a heterologous strain of C. perfringens (Figure 4b,c). We conclude that $\mathrm{rNetB}$ has considerable potential for the development of vaccines against necrotic enteritis. 


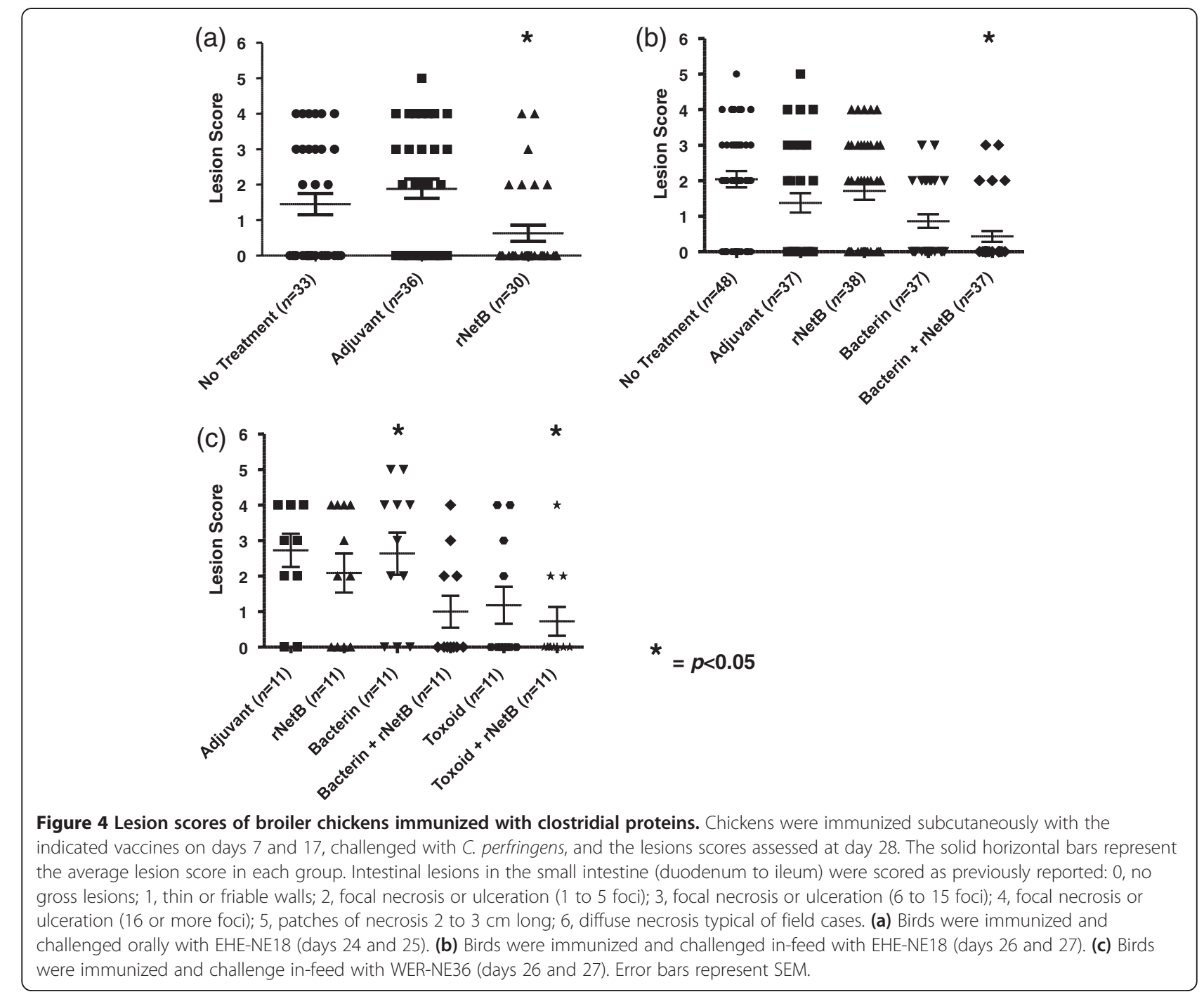

Our results are consistent with other studies that have used cell free toxoids as vaccines [22-24]. Saleh et al. [22] used two C. perfringens isolates from diseased birds (type $\mathrm{A}$ and $\mathrm{C}$ ) to generate three toxoid vaccines (type A, type $C$, and type A plus type C). A significant decrease in disease was observed with all three toxoid vaccines, with the highest protection coming from the combined type $\mathrm{A}$ and $\mathrm{C}$ toxoid. The birds immunised with this toxoid also had higher antibody titres compared to the other toxoid vaccines. Other studies have used toxoids to immunise broiler breeder hens, on the premise that they will develop maternal antibodies that are transferred to the broiler progeny [23,24]. Lovland et al. [24] immunized birds with type A and C cell free toxoid and found that the type $\mathrm{C}$ toxoid protected progeny better than type A. For both toxoids, the level of anti-alpha-toxin IgY antibodies was monitored in the hens and in day old progeny. The antibody titres in vaccinated birds were higher than those in unvaccinated hens and the data suggested a protective effect on the progeny against subclinical necrotic enteritis. However, in their study birds immunised with the type $\mathrm{C}$ toxoid had lower anti-alpha-toxin IgY antibody levels, but gave the better protective effect. This result may be due to the type $\mathrm{C}$ strain also having higher levels of protective antigens. While the NetB status of the strains used to produce the cell-free toxoids in these studies is not known, it is unlikely that the strains that were used produced NetB since both strains were isolated from mammals [24] and there is only one report of a NetB positive C. perfringens being isolated from an animal other than poultry [17].

A similar study by Crouch et al. [23] immunised broiler layer hens from Germany and Italy with the commercially available necrotic enteritis vaccine $\left(\operatorname{NetVax}^{\mathrm{Tx}}\right)$. The safety and efficacy of the $C$. perfringens type A alpha-toxoid was investigated. Again, the NetB status of the strain used to prepare the vaccine was not reported, 
but it was presumably NetB negative as the strain was isolated from cattle. Vaccination resulted in a significant increase in anti-alpha-toxin antibody in the hens and antibodies were detected in the progeny. In contrast to the previous study [24], which detected C. perfringens at greater than $4 \times 10^{4} \mathrm{CFU} / \mathrm{g}$, chicks from $\mathrm{NetVax}^{\text {th }}$ vaccinated hens had no detectable levels of $C$. perfringens. The absence of $C$. perfringens cells in chicks from vaccinated hens suggests that maternal vaccination with NetVax ${ }^{\text {max }}$ reduces the ability of the bacteria to colonise the gastrointestinal tract of chickens.

In our study, the addition of rNetB to bacterin or toxoid preparations from a NetB-producing C. perfringens strain significantly lowered the average lesion score compared to the adjuvant control. Birds immunized with bacterin had low titres of anti-NetB antibody, suggesting that the native NetB protein is in low abundance in these preparations (Figure $3 \mathrm{~b}, \mathrm{c}$ ). In vitro grown cultures contain too little NetB to induce a protective immune response, hence supplementation with exogenous recombinant NetB was required to induce a significant anti-NetB immune response.

A recent study investigated the immunoprotective potential of native culture supernatants from disease and non-disease derived $C$. perfringens isolates [25]. Of the eight strains tested, two supernatants provided full and partial protection (strains $23\left(\mathrm{NetB}^{+}\right)$and $48\left(\mathrm{NetB}^{-}\right)$, respectively). The authors concluded that neither alphatoxin nor NetB were solely immunoprotective. Unfortunately, the sera from the vaccinated birds were not analysed to identify the possible protective antigens, including NetB and alpha-toxin. Our results are in agreement with this study; we confirmed that $\mathrm{rNetB}$ alone was not highly immunoprotective against moderate $C$. perfringens challenge and demonstrated that supplemented rNetB, in conjunction with other antigens, increased the protective response compared to native antigens. This result is not surprising since $C$. perfringens infections are multifactorial and most clostridial vaccines are made up of a cocktail of protective antigens, most often cell free toxoids, rather than a single subunit based vaccine.

Analysis of the convalescent sera from birds challenged with strains EHE-NE18 and WER-NE36 indicated that NetB IgY antibodies were generated during infection, which is in agreement with a study that looked at both alpha-toxin and NetB antibody levels in birds that have been experimentally challenged and birds from natural outbreaks [26]. That study found that challenged birds had a peak anti-NetB IgY antibody level 7 days post-challenge, after which it dramatically dropped back to unchallenged control levels 14 days post-infection. The results from our work and Lee et al. [26] highlight the importance of NetB as a protective antigen and its significance in disease.
In this study rNetB, when used as a subunit vaccine, was protective against oral gavage $C$. perfringens challenge, but not against a more severe challenge. This result is similar to a recent study where birds vaccinated with rNetB had a significant decrease in lesion score compared to the adjuvant control [18]. These authors investigated the role of cellular immunity in protection against C. perfringens/Eimeria maxima co-infection and found that vaccination with ISA 71 VG adjuvant plus recombinant $C$. perfringens antigens reduced transcripts of proinflamatory cytokines in intestinal intraepithelial lymphocytes. Other recombinant antigens including alphatoxin, pyruvate:ferredoxin oxidoreductase, a hypothetical protein, glyceraldehyde-3-phosphate dehydrogenase, fructose 1,6-biphosphate aldolase, endo- $\beta$ - $N$-acetylglucosaminidase, and phosphoglyceromutase have also been shown to significantly protect birds from necrotic enteritis $[7,13,18]$. The role of alpha-toxin in immunity has been studied extensively and it has been shown to confer variable levels of protection [7-9,13,23,24,27]. It is clear that there is a growing body of evidence that other antigens, other than alpha-toxin, can confer protection against disease [11-13,26,28]. NetB can be added to the list of protective antigens against necrotic enteritis [18].

The findings in this study represent the first steps towards the development of an effective vaccine against necrotic enteritis in chickens. The vaccination regime used in the experiments is unlikely to be appropriate for widespread use in broiler chicks because of the multiple handling of individual birds required. A more industry appropriate regime would be to vaccinate the breeder hens, such as used in other studies [23,24] to induce protection in progeny chicks; or vaccine delivery in feed, water, or aerosolized, but that would require sophisticated adjuvant and delivery technology.

In conclusion, we have shown that $\mathrm{rNetB}$ immunization can significantly protect birds against disease, but not against a severe challenge. The greatest protection observed in this study came from birds immunized with cell free toxoid or bacterin supplemented with rNetB; these "enhanced toxoids" may be suitable candidates for use in the poultry industry in the near future.

\section{Competing interests}

ALK, JIR and RJM have a patent on NetB and its use in vaccines: Clostridial Toxin NetB. US Patent no. 8,263,088 B2.

\section{Authors' contributions}

ALK, JIR, RJM conceived and designed the experiments. ALK, RWP, KS, MEF, TLB, XY, RJM performed the experiments and analysed the data. ALK performed the statistical analysis. ALK drafted the paper and RJM and JIR modified and refined it. All authors read and approved the final manuscript.

\section{Acknowledgements}

This research was conducted within the Poultry CRC, established and supported under the Australian Government's Cooperative Research Centres Program. Support was also provided by the Australian Research Council to 
the Australian Research Council Centre of Excellence in Structural and Functional Microbial Genomics at Monash University. We thank the Monash University Protein Production Unit for the preparation of purified rNetB protein.

\section{Author details}

${ }^{1}$ CSIRO Biosecurity Flagship, Australian Animal Health Laboratory, Geelong 3220, Australia. ${ }^{2}$ ARC Centre of Excellence in Structural and Functional Microbial Genomics, Department of Microbiology, Monash University, Clayton 3800, Australia. ${ }^{3}$ Poultry Cooperative Research Centre, Armidale, Australia. ${ }^{4}$ Health Sciences Institute, Federal University of Bahia, Salvador, Brazil.

Received: 14 May 2013 Accepted: 26 June 2013

Published: 16 July 2013

\section{References}

1. Van der Sluis W: Clostridial enteritis is an often underestimated problem. World Poultry 2000, 16:42-43.

2. Van Immerseel F, De Buck J, Pasmans F, Huyghebaert G, Haesebrouck F, Ducatelle R: Clostridium perfringens in poultry: an emerging threat for animal and public health. Avian Pathol 2004, 33:537-549.

3. Dahiya JP, Wilkie DC, Van Kessel AG, Drew MD: Potential strategies for controlling necrotic enteritis in broiler chickens in post-antibiotic era. Anim Feed Sci Technol 2006, 129:60-88.

4. Grave K, Kaldhusdal MC, Kruse H, Harr LMF, Flatlandsmo K: What has happened in norway after the ban of avoparcin? Consumption of antimicrobials by poultry. Prev Vet Med 2004, 62:59-72.

5. Walker PD: Bacterial vaccines: old and new, veterinary and medical. Vaccine 1992, 10:977-990.

6. Parish WE: Necrotic enteritis in the fowl (Gallus gallus domesticus). I. Histopathology of the disease and isolation of a strain of Clostridium welchii. J Comp Pathol 1961, 71:377-393.

7. Cooper KK, Trinh HT, Songer JG: Immunization with recombinant alpha toxin partially protects broiler chicks against experimental challenge with Clostridium perfringens. Vet Microbiol 2009, 133:92-97.

8. Hoang TH, Hong HA, Clark GC, Titball RW, Cutting SM: Recombinant Bacillus subtilis expressing the Clostridium perfringens alpha toxoid is a candidate orally delivered vaccine against necrotic enteritis. Infect Immun 2008, 76:5257-5265.

9. Zekarias B, Mo H, Curtiss R: Recombinant attenuated Salmonella enterica serovar typhimurium expressing the carboxy-terminal domain of alpha toxin from Clostridium perfringens induces protective responses against necrotic enteritis in chickens. Clin Vaccine Immunol 2008, 15:805-816.

10. Keyburn AL, Sheedy SA, Ford ME, Williamson MM, Awad MM, Rood Jl, Moore RJ: Alpha-toxin of Clostridium perfringens is not an essential virulence factor in necrotic enteritis in chickens. Infect Immun 2006, 74:6496-6500

11. Thompson DR, Parreira VR, Kulkarni RR, Prescott JF: Live attenuated vaccine-based control of necrotic enteritis of broiler chickens. Vet Microbiol 2006, 113:25-34.

12. Kulkarni RR, Parreira VR, Sharif S, Prescott JF: Clostridium perfringens antigens recognized by broiler chickens immune to necrotic enteritis. Clin Vaccine Immunol 2006, 13:1358-1362.

13. Kulkarni RR, Parreira VR, Sharif S, Prescott JF: Immunization of broiler chickens against Clostridium perfringens-induced necrotic enteritis. Clin Vaccine Immunol 2007, 14:1070-1077.

14. Keyburn AL, Boyce JD, Vaz P, Bannam TL, Ford ME, Parker D, Di Rubbo A, Rood JI, Moore RJ: NetB, a new toxin that is associated with avian necrotic enteritis caused by Clostridium perfringens. PLoS Pathog 2008, 4:e26.

15. Nowell VJ, Poppe C, Parreira VR, Jiang Y-F, Reid-Smith R, Prescott JF: Clostridium perfringens in retail chicken. Anaerobe 2010, 16:314-315.

16. Keyburn AL, Yan X-X, Bannam TL, Van Immerseel F, Rood JI, Moore RJ: Association between avian necrotic enteritis and Clostridium perfringens strains expressing NetB toxin. Vet Res 2010, 41:21.

17. Martin TG, Smyth JA: Prevalence of netB among some clinical isolates of Clostridium perfringens from animals in the United States. Vet Microbiol 2009, 136:202-205.

18. Jang SI, Lillehoj HS, Lee S-H, Lee KW, Lillehoj EP, Hong YH, An D-J, Jeong W, Chun J-E, Bertrand F, Dupuis L, Deville S, Arous JB: Vaccination with
Clostridium perfringens recombinant proteins in combination with Montanide $^{\mathrm{TM}}$ ISA $71 \mathrm{VG}$ adjuvant increases protection against experimental necrotic enteritis in commercial broiler chickens. Vaccine 2012, 30:5401-5406.

19. Prowse S: A new adjuvant. ANZCCART 2000, 13:7.

20. Cooper KK, Songer JG: Virulence of Clostridium perfringens in an experimental model of poultry necrotic enteritis. Vet Microbiol 2010, 142:323-328.

21. Frey A, Di Canzio J, Zurakowski D: A statistically defined endpoint titer determination method for immunoassays. J Immunol Methods 1998, 221:35-41.

22. Saleh N, Fathalla SI, Nabil R, Mosaad AA: Clinicopathological and immunological studies on toxoids vaccine as a successful alternative in controlling clostridial infection in broilers. Anaerobe 2011, 17:426-430.

23. Crouch CF, Withanage GSK, de Haas V, Etoré F, Francis MJ: Safety and efficacy of a maternal vaccine for the passive protection of broiler chicks against necrotic enteritis. Avian Pathol 2010, 39:489-497.

24. Lovland A, Kaldhusdal M, Redhead K, Skjerve E, Lillehaug A: Maternal vaccination against subclinical necrotic enteritis in broilers. Avian Pathol 2004, 33:83-92.

25. Lanckriet A, Timbermont L, Eeckhaut V, Haesebrouck F, Ducatelle R, Van Immerseel F: Variable protection after vaccination of broiler chickens against necrotic enteritis using supernatants of different Clostridium perfringens strains. Vaccine 2010, 28:5920-5923.

26. Lee KW, Lillehoj HS, Park MS, Jang SI, Ritter GD, Hong YH, Jeong W, Jeoung HY, An DJ, Lillehoj EP: Clostridium perfringens a-toxin and NetB toxin antibodies and their possible role in protection against necrotic enteritis and gangrenous dermatitis in broiler chickens. Avian Dis 2012, 56:230-233

27. Kulkarni RR, Parreira VR, Jiang Y-F, Prescott JF: A live oral recombinant Salmonella enterica serovar typhimurium vaccine expressing Clostridium perfringens antigens confers protection against necrotic enteritis in broiler chickens. Clin Vaccine Immunol 2010, 17:205-214.

28. Kulkarni RR, Parreira VR, Sharif S, Prescott JF: Oral immunization of broiler chickens against necrotic enteritis with an attenuated Salmonella vaccine vector expressing Clostridium perfringens antigens. Vaccine 2008 26:4194-4203.

doi:10.1186/1297-9716-44-54

Cite this article as: Keyburn et al:: Vaccination with recombinant NetB toxin partially protects broiler chickens from necrotic enteritis. Veterinary Research 2013 44:54.

\section{Submit your next manuscript to BioMed Central and take full advantage of:}

- Convenient online submission

- Thorough peer review

- No space constraints or color figure charges

- Immediate publication on acceptance

- Inclusion in PubMed, CAS, Scopus and Google Scholar

- Research which is freely available for redistribution

Submit your manuscript at www.biomedcentral.com/submit
C Biomed Central 